\title{
On the $k$-free divisor problem (II)
}

by

Jun Furuya (Okinawa) and Wenguang Zhai (Jinan)

1. Introduction. Let $d(n)$ denote the divisor function. Dirichlet first proved that the error term

$$
\Delta(x):=\sum_{n \leq x}^{\prime} d(n)-x \log x-(2 \gamma-1) x, \quad x \geq 2,
$$

satisfies $\Delta(x)=O\left(x^{1 / 2}\right)$, where $\sum_{n \leq x}^{\prime}$ means that the term for $n=x$ should be halved when $x$ is an integer. The exponent $1 / 2$ was improved by many authors. The latest result is due to Huxley [4], who proved that

$$
\Delta(x) \ll x^{131 / 416}(\log x)^{26957 / 8320} .
$$

It is conjectured that

$$
\Delta(x)=O\left(x^{1 / 4+\varepsilon}\right),
$$

which is supported by the classical mean-square result

$$
\int_{1}^{T} \Delta^{2}(x) d x=\frac{(\zeta(3 / 2))^{4}}{6 \pi^{2} \zeta(3)} T^{3 / 2}+O\left(T \log ^{5} T\right)
$$

proved by Tong [12].

Let $k \geq 2$ denote a fixed integer. An integer $n$ is called $k$-free if $p^{k}$ does not divide $n$ for any prime $p$. Let $d^{(k)}(n)$ denote the number of $k$-free divisors of the positive integer $n$ and define

$$
D^{(k)}(x):=\sum_{n \leq x}^{\prime} d^{(k)}(n) .
$$

2000 Mathematics Subject Classification: Primary 11N37.

Key words and phrases: Dirichlet divisor problem, $k$-free divisor problem.

J. Furuya is supported by a Grant-in-Aid for Scientific Research from the Ministry of Education, Science, Sports and Culture, Japan (No. 18740022). W. G. Zhai is supported by National Natural Science Foundation of China (Grant No. 10771127) and National Natural Science Foundation of Shandong Province (Grant No. Y2006A31). 
Then the expected asymptotic formula for $D^{(k)}(x)$ is

$$
D^{(k)}(x)=C_{1}^{(k)} x \log x+C_{2}^{(k)} x+\Delta^{(k)}(x),
$$

where $C_{1}^{(k)}, C_{2}^{(k)}$ are two constants, and $\Delta^{(k)}(x)$ is the error term. In 1874 Mertens [7] proved that $\Delta^{(2)}(x) \ll x^{1 / 2} \log x$. In 1932 Perron [9] proved that

$$
\Delta^{(k)}(x) \ll \begin{cases}x^{1 / 2} & \text { if } k=2, \\ x^{1 / 3} & \text { if } k=3, \\ x^{33 / 100} & \text { if } k \geq 4 .\end{cases}
$$

For $k=2,3$, it is very difficult to improve the exponent $1 / k$ in the bound $\Delta^{(k)}(x) \ll x^{1 / k}$, unless we have substantial progress in the study of the zerofree region of $\zeta(s)$. Therefore it is reasonable to get better improvements by assuming the truth of the Riemann Hypothesis (RH). Such results have been given in $[1,2,6,8,10,11]$. In particular, in [2] R. C. Baker proved $\Delta^{(2)}(x) \ll x^{4 / 11+\varepsilon}$ and in $[6]$ Kumchev proved $\Delta^{(3)}(x) \ll x^{27 / 85+\varepsilon}$ under RH. For $k \geq 4$, it is easy to show that if $\Delta(x) \ll x^{\alpha}$, then $\Delta^{(k)}(x) \ll x^{\alpha} \log x$.

We believe that the estimate

$$
\Delta^{(k)}(x) \ll x^{1 / 4+\varepsilon}
$$

is true for any $k \geq 2$, which is an analogue of (1.1). For $k \geq 4$ the conjecture (1.4) is partly supported by the asymptotic formula

$$
\int_{1}^{T}\left|\Delta^{(k)}(x)\right|^{2} d x=\frac{B_{k}}{6 \pi^{2}} T^{3 / 2}+ \begin{cases}O\left(T^{3 / 2} e^{-c \delta(T)}\right) & \text { for } k=4, \\ O\left(T^{\delta_{k}+\varepsilon}\right) & \text { for } k \geq 5,\end{cases}
$$

proved in [3], where $c>0$ is an absolute constant and

$$
\begin{aligned}
& B_{k}:=\sum_{m=1}^{\infty} g_{k}^{2}(m) m^{-3 / 2}, \quad g_{k}(m):=\sum_{m=n l^{k}} \mu(l) d(n) l^{k / 2}, \\
& \delta(u):=(\log u)^{3 / 5}(\log \log u)^{-1 / 5}, \\
& \delta_{5}:=29 / 20, \quad \delta_{k}:=3 / 2-1 / 2 k+1 / k^{2} \quad(k \geq 6) .
\end{aligned}
$$

The approach in [3] fails for $k=3$ and gives only a weak result for $k=4$. However, if $\mathrm{RH}$ is true, we can do much better. In this short note, we shall prove the following

THEOREM. If $R H$ is true, then

$$
\int_{1}^{T}\left|\Delta^{(k)}(x)\right|^{2} d x=\frac{B_{k}}{6 \pi^{2}} T^{3 / 2}+O\left(T^{3 / 2-\eta_{k}+\varepsilon}\right)
$$

with $\eta_{k}:=(k-2) /(12 k-8)(k=3,4,5,6)$, where the implied constant depends only on $\varepsilon$. 
Corollary. If $R H$ is true, then

$$
\Delta^{(3)}(x)=\Omega\left(x^{1 / 4}\right) .
$$

Acknowledgements. The authors deeply thank the referee for valuable suggestions.

\section{Proof of Theorem}

2.1. Mean square of $\Delta_{2, y}^{(k)}(x)$. Suppose $\mathrm{RH}$ is true. It is obvious that

$$
\sum_{n=1}^{\infty} \frac{d^{(k)}(n)}{n^{s}}=\frac{\zeta^{2}(s)}{\zeta(k s)} \quad(\Re s>1)
$$

which implies that

$$
d^{(k)}(n)=\sum_{n=l^{k} m} \mu(l) d(m) .
$$

Let $y>2$ be a parameter. Define

$$
d_{1, y}^{(k)}(n):=\sum_{\substack{n=l^{k} m \\ l \leq y}} \mu(l) d(m), \quad d_{2, y}^{(k)}(n):=\sum_{\substack{n=l^{k} m \\ l>y}} \mu(l) d(m) .
$$

Then

$$
d^{(k)}(n)=d_{1, y}^{(k)}(n)+d_{2, y}^{(k)}(n) .
$$

It is easy to see that for $\Re s>1$ we have

$$
\sum_{n=1}^{\infty} \frac{d_{2, y}^{(k)}(n)}{n^{s}}=\zeta^{2}(s) f_{y}(k s)
$$

where $f_{y}(s):=\sum_{l>y} \mu(l) / l^{s}$. It is well-known that under $\mathrm{RH}$, the function $f_{y}(s)$ can be analytically continued to $\Re s>1 / 2$ and that uniformly in the strip $1 / 2+\varepsilon<\Re s \leq 1$ the estimate

$$
f_{y}(s) \ll y^{1 / 2-\sigma+\varepsilon}(1+|t|)^{\varepsilon}
$$

holds.

Let

$$
D_{i, y}^{(k)}(x):=\sum_{n \leq x}^{\prime} d_{i, y}^{(k)}(n) \quad(i=1,2)
$$

Then

$$
D^{(k)}(x)=D_{1, y}^{(k)}(x)+D_{2, y}^{(k)}(x) .
$$

Note that here $y$ is independent of $x$. We have $D_{2, y}^{(k)}(x) \equiv 0$ when $y>x^{1 / k}$. 
For $D_{1, y}^{(k)}(x)$, we have

$$
\begin{aligned}
D_{1, y}^{(k)}(x) & =\sum_{l \leq y} \mu(l) \sum_{m \leq x / l^{k}}^{\prime} d(m) \\
& =\sum_{l \leq y} \mu(l)\left\{\frac{x}{l^{k}} \log \frac{x}{l^{k}}+(2 \gamma-1) \frac{x}{l^{k}}+\Delta\left(\frac{x}{l^{k}}\right)\right\} \\
& =\operatorname{Res}_{s=1}\left(\zeta^{2}(s) \frac{x^{s}}{s} \sum_{l \leq y} \frac{\mu(l)}{l^{s}}\right)+\sum_{l \leq y} \mu(l) \Delta\left(\frac{x}{l^{k}}\right) .
\end{aligned}
$$

By Perron's formula we know that

$$
D_{2, y}^{(k)}(x)=\frac{1}{2 \pi i} \int_{2-i \infty}^{2+i \infty} \zeta^{2}(s) \frac{x^{s}}{s} f_{y}(k s) d s .
$$

Moving the line of integration in (2.5) to some $c<1$ (but close to 1 ), by the residue theorem we get

$$
D_{2, y}^{(k)}(x)=\operatorname{Res}_{s=1}\left(\zeta^{2}(s) \frac{x^{s}}{s} f_{y}(k s)\right)+\frac{1}{2 \pi i} \int_{c-i \infty}^{c+i \infty} \zeta^{2}(s) \frac{x^{s}}{s} f_{y}(k s) d s .
$$

Let

$$
\Delta_{2, y}^{(k)}(x):=\sum_{n \leq x}^{\prime} d_{2, y}^{(k)}(n)-\operatorname{Res}_{s=1}\left(\zeta^{2}(s) \frac{x^{s}}{s} f_{y}(k s)\right)
$$

Then

$$
\Delta_{2, y}^{(k)}(x)=\frac{1}{2 \pi i} \int_{c-i \infty}^{c+i \infty} \zeta^{2}(s) \frac{x^{s}}{s} f_{y}(k s) d s .
$$

Since $\zeta^{2}(s) f_{y}(k s) s^{-1} \rightarrow 0$ uniformly in the strip $1 / 4<\Re s<1$ when $|t| \rightarrow \infty,(2.7)$ is true for any $1 / 4<c<1$. Replacing in $(2.7) x$ by $1 / x$, taking $c=1 / 4+\varepsilon$ and then using Parseval's identity (see for example, (A.5) of Ivić [5]) we get

$$
\int_{0}^{\infty} \frac{\left|\Delta_{2, y}^{(k)}(x)\right|^{2}}{x^{3 / 2+2 \varepsilon}} d x=\frac{1}{2 \pi} \int_{-\infty}^{\infty} \frac{|\zeta(1 / 4+\varepsilon+i t)|^{4}\left|f_{y}(k(1 / 4+\varepsilon+i t))\right|^{2}}{|1 / 4+\varepsilon+i t|^{2}} d t
$$

From (2.2) we have

$$
\left|f_{y}(k(1 / 4+\varepsilon+i t))\right|^{2} \ll y^{1-k / 2-2 k \varepsilon+2 \varepsilon}(1+|t|)^{2 \varepsilon} .
$$

Under $\mathrm{RH}$ we have, for any $0 \leq \sigma \leq 1 / 2$,

$$
\zeta(\sigma+i t) \ll(1+|t|)^{1 / 2-\sigma+\varepsilon / 4} .
$$

Thus

$$
|\zeta(1 / 4+\varepsilon+i t)|^{4} \ll(1+|t|)^{1-3 \varepsilon},
$$


which combined with (2.8) and (2.9) implies

$$
\int_{0}^{\infty} \frac{\left|\Delta_{2, y}^{(k)}(x)\right|^{2}}{x^{3 / 2+2 \varepsilon}} d x \ll y^{1-k / 2-2 k \varepsilon+2 \varepsilon} \int_{-\infty}^{\infty}(1+|t|)^{-1-\varepsilon} d t \ll y^{1-k / 2} .
$$

Hence for any $M>2$ we have

$$
\int_{M}^{2 M} \frac{\left|\Delta_{2, y}^{(k)}(x)\right|^{2}}{x^{3 / 2+2 \varepsilon}} d x \ll y^{1-k / 2}
$$

so

$$
\int_{M}^{2 M}\left|\Delta_{2}^{(k)}(x)\right|^{2} d x \ll M^{3 / 2+\varepsilon} y^{1-k / 2} .
$$

2.2. Completion of proof. Suppose $T \geq 10$ is large. It suffices to evaluate the integral $\int_{T}^{2 T}\left|\Delta^{(k)}(x)\right|^{2} d x$. From (2.3)-(2.7) we have

$$
\Delta^{(k)}(x)=\Delta_{1, y}^{(k)}(x)+\Delta_{2, y}^{(k)}(x)
$$

where

$$
\Delta_{1, y}^{(k)}(x):=\sum_{l \leq y} \mu(l) \Delta\left(x / l^{k}\right) .
$$

Let $T^{\varepsilon} \ll y \ll T^{1 / 4-\varepsilon}$ and $T^{\varepsilon} \ll z \ll T^{1-\varepsilon}$ be two parameters to be determined later. Let

$$
\Delta_{1}(u):=\frac{u^{1 / 4}}{\sqrt{2} \pi} \sum_{n \leq z} \frac{d(n)}{n^{3 / 4}} \cos (4 \pi \sqrt{n u}-\pi / 4), \quad \Delta_{2}(u ; z):=\Delta(u)-\Delta_{1}(u) .
$$

Then we can write

$$
\Delta_{1, y}^{(k)}(x)=R_{1}^{(k)}(x)+R_{2}^{(k)}(x),
$$

where

$$
\begin{aligned}
& R_{1}^{(k)}(x):=\frac{x^{1 / 4}}{\sqrt{2} \pi} \sum_{l \leq y} \frac{\mu(l)}{l^{k / 4}} \sum_{n \leq z} \frac{d(n)}{n^{3 / 4}} \cos \left(4 \pi \sqrt{\frac{n x}{l^{k}}}-\frac{\pi}{4}\right), \\
& R_{2}^{(k)}(x):=\sum_{l \leq y} \mu(l) \Delta_{2}\left(\frac{x}{l^{k}} ; z\right) .
\end{aligned}
$$

Taking $z=T^{1-\varepsilon}$ we deduce from (3.3) of [3] that

$$
\begin{aligned}
\int_{T}^{2 T}\left|R_{2}^{(k)}(x)\right|^{2} d x & \ll \begin{cases}T^{3 / 2} z^{-1 / 2} y^{1 / 2} \log ^{4} T+T y^{2} \log ^{6} T & \text { if } k=3, \\
T^{3 / 2} z^{-1 / 2} \log ^{5} T+T y^{2} \log ^{6} T & \text { if } k \geq 4,\end{cases} \\
& \ll T y^{2} \log ^{6} T \quad(k \geq 3) .
\end{aligned}
$$


Now we consider the mean square of $R_{1}^{(k)}(x)$. By the elementary formula

$$
\cos u \cos v=\frac{1}{2}(\cos (u-v)+\cos (u+v))
$$

we may write

$$
\begin{aligned}
\left|R_{1}^{(k)}(x)\right|^{2}= & \frac{x^{1 / 2}}{2 \pi^{2}} \sum_{l_{1}, l_{2} \leq y} \frac{\mu\left(l_{1}\right) \mu\left(l_{2}\right)}{\left(l_{1} l_{2}\right)^{k / 4}} \sum_{n_{1}, n_{2} \leq z} \frac{d\left(n_{1}\right) d\left(n_{2}\right)}{\left(n_{1} n_{2}\right)^{3 / 4}} \\
& \times \cos \left(4 \pi \sqrt{\frac{n_{1} x}{l_{1}^{k}}}-\frac{\pi}{4}\right) \cos \left(4 \pi \sqrt{\frac{n_{2} x}{l_{2}^{k}}}-\frac{\pi}{4}\right) \\
= & S_{1}(x)+S_{2}(x)+S_{3}(x),
\end{aligned}
$$

where

$$
\begin{aligned}
& S_{1}(x)=\frac{x^{1 / 2}}{4 \pi^{2}} \sum_{\substack{l_{1}, l_{2} \leq y ; n_{1}, n_{2} \leq z \\
n_{1} l_{2}^{k}=n_{2} l_{1}^{k}}} \frac{\mu\left(l_{1}\right) \mu\left(l_{2}\right)}{\left(l_{1} l_{2}\right)^{k / 4}} \frac{d\left(n_{1}\right) d\left(n_{2}\right)}{\left(n_{1} n_{2}\right)^{3 / 4}}, \\
& S_{2}(x) \\
& \quad=\frac{x^{1 / 2}}{4 \pi^{2}} \sum_{\substack{l_{1}, l_{2} \leq y ; n_{1}, n_{2} \leq z \\
n_{1} l_{2}^{k} \neq n_{2} l_{1}^{k}}} \frac{\mu\left(l_{1}\right) \mu\left(l_{2}\right)}{\left(l_{1} l_{2}\right)^{k / 4}} \frac{d\left(n_{1}\right) d\left(n_{2}\right)}{\left(n_{1} n_{2}\right)^{3 / 4}} \cos \left(4 \pi \sqrt{x}\left(\sqrt{\frac{n_{1}}{l_{1}^{k}}}-\sqrt{\frac{n_{2}}{l_{2}^{k}}}\right)\right), \\
& \quad=\frac{x^{1 / 2}}{4 \pi^{2}} \sum_{S_{l_{1}, l_{2} \leq y ; n_{1}, n_{2} \leq z}} \frac{\mu\left(l_{1}\right) \mu\left(l_{2}\right)}{\left(l_{1} l_{2}\right)^{k / 4}} \frac{d\left(n_{1}\right) d\left(n_{2}\right)}{\left(n_{1} n_{2}\right)^{3 / 4}} \sin \left(4 \pi \sqrt{x}\left(\sqrt{\frac{n_{1}}{l_{1}^{k}}}+\sqrt{\frac{n_{2}}{l_{2}^{k}}}\right)\right) .
\end{aligned}
$$

From (3.7) and (4.4) of [3] we have

$$
\int_{T}^{2 T} S_{1}(x) d x=\frac{B_{k}}{4 \pi^{2}} \int_{T}^{2 T} x^{1 / 2} d x+O\left(T^{3 / 2+\varepsilon} y^{-1 / 2+1 / k}\right) .
$$

From (3.8) and (5.10) of [3] we get

$$
\int_{T}^{2 T} S_{2}(x) d x \ll T^{1+\varepsilon} y^{2}+T^{1+(k+1) / 3 k+\varepsilon} .
$$

From (3.9) of [3] we have

$$
\int_{T}^{2 T} S_{3}(x) d x \ll T y^{2} \log ^{4} T .
$$

From (2.14)-(2.17) we obtain 


$$
\begin{aligned}
\int_{T}^{2 T}\left|R_{1}^{(k)}(x)\right|^{2} d x= & \frac{B_{k}}{4 \pi^{2}} \int_{T}^{2 T} x^{1 / 2} d x+O\left(T^{3 / 2+\varepsilon} y^{-1 / 2+1 / k}\right) \\
& +O\left(T^{1+\varepsilon} y^{2}+T^{1+(k+1) / 3 k+\varepsilon}\right) .
\end{aligned}
$$

From (2.13), (2.18) and the Cauchy inequality we get

$$
\int_{T}^{2 T} R_{1}^{(k)}(x) R_{2}^{(k)}(x) d x \ll T^{5 / 4} y \log ^{3} T .
$$

From (2.13), (2.18) and (2.19) we get

$$
\begin{aligned}
\int_{T}^{2 T}\left|\Delta_{1, y}^{(k)}(x)\right|^{2} d x= & \frac{B_{k}}{4 \pi^{2}} \int_{T}^{2 T} x^{1 / 2} d x+O\left(T^{3 / 2+\varepsilon} y^{-1 / 2+1 / k}\right) \\
& +O\left(T^{5 / 4} y \log ^{3} T+T^{1+(k+1) / 3 k+\varepsilon}\right),
\end{aligned}
$$

which combining (2.10) with $M=T$ gives

$$
\int_{T}^{2 T} \Delta_{1, y}^{(k)}(x) \Delta_{2, y}^{(k)}(x) d x \ll T^{3 / 2+\varepsilon} y^{-(k-2) / 4} .
$$

From $(2.20),(2.21)$ and (2.10) with $M=T$ and then taking $y=T^{k / 2(3 k-2)}$ we get

$$
\begin{aligned}
\int_{T}^{2 T}\left|\Delta^{(k)}(x)\right|^{2} d x= & \frac{B_{k}}{4 \pi^{2}} \int_{T}^{2 T} x^{1 / 2} d x+O\left(T^{3 / 2+\varepsilon} y^{-1 / 2+1 / k}\right) \\
& +O\left(T^{5 / 4} y \log ^{3} T+T^{1+(k+1) / 3 k+\varepsilon}\right) \\
= & \frac{B_{k}}{4 \pi^{2}} \int_{T}^{2 T} x^{1 / 2} d x+O\left(T^{3 / 2-\eta_{k}+\varepsilon}\right)
\end{aligned}
$$

where $\eta_{k}$ was defined in the Theorem upon noting that $(k-2) / 4 \geq 1 / 2-1 / k$. Hence our Theorem follows from a splitting argument.

\section{References}

[1] R. C. Baker, The square-free divisor problem, Quart. J. Math. Oxford Ser. (2) 45 (1994), 269-277.

[2] - , The square-free divisor problem II, ibid. 47 (1996), 133-146.

[3] J. Furuya and W. G. Zhai, On the k-free divisor problem, Acta Arith. 123 (2006), $267-287$.

[4] M. N. Huxley, Exponential sums and lattice points III, Proc. London Math. Soc. 87 (2003), 591-609.

[5] A. Ivić, The Riemann Zeta-Function, Wiley, 1985.

[6] A. Kumchev, The k-free divisor problem, Monatsh. Math. 129 (2000), 321-327. 
[7] F. Mertens, Über einige asymptotische Gesetze der Zahlentheorie, J. Reine Angew. Math. 77 (1874), 289-338.

[8] W. G. Nowak and M. Schmeier, Conditional asymptotic formulae for a class of arithmetic functions, Proc. Amer. Math. Soc. 103 (1988), 713-717.

[9] O. Perron, Über einen asymptotischen Ausdruck, Acta Math. 59 (1932), 89-97.

[10] B. Saffari, Sur le nombre de diviseurs "r-libres" d'un entier, et sur les points à coordonnées entières dans certaines régions du plan, C. R. Acad. Sci. Paris Sér. A-B 266 (1968), A601-A603.

[11] V. Siva Rama Prasad and D. Suryanarayana, The number of $k$-free divisors of an integer, Acta Arith. 17 (1970/71), 345-354.

[12] K. C. Tong, On divisor problems III, Acta Math. Sinica 6 (1956), 515-541.

Department of Integrated Arts and Science

Okinawa National College of Technology

Nago, Okinawa, 905-2192

Japan

E-mail: jfuruya@okinawa-ct.ac.jp
School of Mathematical Sciences

Shandong Normal University

Jinan, Shandong, 250014

P.R. China

E-mail: zhaiwg@hotmail.com

Received on 25.7.2007

and in revised form on 10.1.2008 